\title{
Geographical origin discrimination and polysaccharides quantitative analysis of Radix codonopsis with micro near-infrared spectrometer engine
}

\author{
Jiayue Wang*, Tongtong $\mathrm{Li}^{*}$, Hailong Yang ${ }^{\dagger}$, Tian $\mathrm{Hu}^{*}$, \\ Lei Nie*, Fei Wang*, Manel Alcalà ${ }^{\star}, \|$ and Hengchang Zang*, ${ }^{*}, \|$ \\ *School of Pharmaceutical Sciences, Shandong University \\ Wenhuaxi Road 44, Jinan 250012, China \\ ${ }^{\dagger}$ FOSS (Beijing) Science Technology and Trading Co., Ltd \\ Zhong Guan Cun South Street, Beijing 100081, China \\ \$Department of Chemistry, Faculty of Sciences \\ Universitat Autònoma de Barcelona \\ 08193 Bellaterra, Barcelona, Spain \\ \$manel.alcala@uab.cat \\ 『zanghcw@126.com
}

Received 13 May 2017

Accepted 24 July 2017

Published 10 August 2017

\begin{abstract}
At present, Tradition Chinese Medicine (TCM) industry in China is in the stage from the empirical development to industrial production. Near infrared (NIR) spectroscopy has been widely used in the quality control of TCM's modernization with its characteristics including rapidness, nondestruction, simplicity, economy, and so on. In this study, as one type of a portable micro NIR spectrometer, Micro NIR 1700 was used to establish the qualitative models for identification of geographical region and authenticity of Radix codonopsis based on discriminant analysis (DA) method. Both of the DA models had better predictive ability with $100 \%$ accuracy. In addition, a method for rapid quantitative analysis of polysaccharide in Radix codonopsis was also developed based on Micro NIR 1700 spectrometer with partial least-squares (PLS) algorithm. In the PLS calibration model, the NIR spectra of samples were pretreated with different preprocessing methods and the spectral region was selected with different variable selection methods as well. The performance of the final PLS model was evaluated according to correlation coefficient of calibration $\left(R_{c}\right)$, correlation coefficient of prediction $\left(R_{p}\right)$, root mean squared error of cross validation (RMSECV), and root mean squared of prediction (RMSEP). The values of $R_{c}$, $R_{p}$, RMSECV, and RMSEP were $0.9775,0.9602,2.496$, and $2.734 \mathrm{~g} / \mathrm{mL}$, respectively. This work demonstrated that micro infrared spectrometer could be more convenient and rapid for quality
\end{abstract}

\|Corresponding authors.

This is an Open Access article published by World Scientific Publishing Company. It is distributed under the terms of the Creative Commons Attribution 4.0 (CC-BY) License. Further distribution of this work is permitted, provided the original work is properly cited. 
control of Radix codonopsis, and the presented models would be a useful reference for quality control of other similar raw materials of TCM.

Keywords: Tradition Chinese Medicine; Micro NIR 1700 spectrometer; Radix codonopsis.

\section{Introduction}

Currently, the Tradition Chinese Medicine (TCM) industry in China is in the stage from the empirical development to industrial production. Many issues have occurred in the process, such as the complex sources of raw materials, uneven quality levels, the lack of strict quality control standards as well as scientific techniques, and so on. These issues hinder the development of TCM industry. Therefore, we should apply multi-disciplinary knowledge to make TCM production technology normalized, standardized, scientific, and systematized. ${ }^{1}$ It is particularly important for raw material of TCM to establish rapid quality evaluation system because it is the starting point of TCM preparation production, and reliable quality of raw material is a crucial guarantee to the final product of TCM.

Radix codonopsis is the root of Codonopsis pilosula (Franch.) Nannf., Codonopsis pilosula Nannf. var. modesta (Nannf.) L. T Shen, or Codonopsis tangshen Oliv., belonging to the family Campanulaceae. It has been used for benefiting stomach and spleen, promoting the production of blood and body fluid. Besides, it also has the function of antitumor, antimicrobial, antioxidant, and so on. ${ }^{2}$ Radix codonopsis is a kind of important raw material for numerous TCM preparations including all nourishing bolus, gynecologic Qianjin tablets, and so on. Additionally, owing to Radix codonopsis contains rich carbohydrate, amino acid, vitamin, and mineral composition, it has also been utilized into many healthcare products.

C. pilosula is mainly cultivated in the Northeast of China, Shanxi Province, Gansu Province, Henan Province, and Sichuan Province. The chemical composition and content in raw material of TCM planted in different areas vary greatly with the condition of soil, water quality, climate, and other factors, which can affect their curative effect and clinical application. Besides, TCM raw material has counterfeiting phenomenon in medicinal market due to profit-driven. Traditional quality control methods, including empirical method and microscopy method, highly depend on personal skills and cannot achieve rapid, objective, and accurate analysis. Therefore, an accurate and rapid method is necessary to develop for the identification of geographical origins and authenticity of C. pilosula. $C$. pilosula contains a high content of polysaccharides, ${ }^{3,4}$ so many literatures about polysaccharides were investigated. Polysaccharide is one of the most important active ingredients of $C$. pilosula, possessing antimicrobial, antitumor, and antioxidant activities. ${ }^{5-7}$ At present, the most usual way to determine the content of polysaccharides is phenol-sulfuric acid method. ${ }^{8}$ However, this method is not only complicated but also time-consuming. Thus, it is essential to establish a convenient method for rapid determination of the content of polysaccharides in C. pilosula for quality evaluation.

As a booming analytical method, ${ }^{9}$ near infrared spectroscopy (NIRS) has been widely applied in many fields, such as petroleum chemical industry, agriculture and food business, tobacco industry, and pharmacy ${ }^{10-13}$ due to its characteristics ${ }^{14}$ including rapidness, nondestruction, simplicity, low cost, and so on. In the field of TCM, NIRS has been widely employed in both qualitative analysis and quantitative determination. ${ }^{15,16}$ Meanwhile, it can serve as a kind of process analysis technology in on-line quality control of TCM manufacture. ${ }^{17,18}$ NIR spectrometer is generally expensive and bulky for routine analysis, which is not suitable for rapid onsite analysis in pharmaceutical company. Micro NIR 1700 spectrometer (VIAVI, USA) is a new and portable NIR spectrometer, following particular features such as lightweight, USB power supply or battery, etc. ${ }^{19}$ When compared with laboratoryscale instrument, it guarantees the accuracy of results but also with the advantages of portability and low cost. With the aforementioned characteristics, it is becoming a new research highlight. Pan et $a l .{ }^{20}$ showed variable importance in projection assignment of particle size and lobetyolin content of C. pilosula in NIR model development. Li et al. ${ }^{21}$ showed discrimination of the geographical origin of C. pilosula using NIR diffuse reflection spectroscopy coupled with random forests and $k$-nearest neighbor 
methods. The aim of the present research is to apply Micro NIR 1700 spectrometer to establish the qualitative model for quickly discriminating geographical origins ${ }^{22}$ and the counterfeit of $C$. pilosula with discriminant analysis (DA) method. ${ }^{23,24}$ Meanwhile, the calibration model was constructed for quantitative analysis of polysaccharide content in C. pilosula by Micro NIR 1700 spectrometer and partial least square (PLS) regression. To the best of our knowledge, there was no similar report about the qualitative discrimination and quantitative analysis of polysaccharide in C. pilosula with micro NIR spectrometer engine.

\section{Materials and Methods}

\subsection{Materials}

In this research, a total of 102 batches of C. pilosula were collected from the medicinal market of Bozhou (Anhui Province, China). The information of samples was shown in Table 1.

\subsection{Methods}

\subsubsection{Spectral collection}

First, samples of $C$. pilosula were crushed and then screened through mesh size of 60 and dried at $60^{\circ}$ for $24 \mathrm{~h}$. The diffuse reflectance spectra of 102 samples were acquired by using a Micro NIR 1700 spectrometer (VIAVI, USA) equipped with a dualintegrated vacuum tungsten lamp, a thin-film linearly variable filter, and a 128-line element uncooled indium gallium arsenide (InGaAs) diode array. The structure of Micro NIR 1700 spectrometer was shown in Fig. 1.
The reference measurement was performed with a $100 \%$ Spectralon $^{\mathrm{TM}}$ reference standard before sample measurement. The wavelength ranges from $908 \mathrm{~nm}$ to $1676 \mathrm{~nm}$. The integration time of Micro NIR was set to $5000 \mathrm{~ms}$, and each spectrum was accumulated of 100 scans. Each sample was scanned three times and then averaged for analysis. Spectra were recorded at room temperature and the humidity was maintained at $40 \%$.

\subsubsection{Software}

Qualitative model for quickly detecting the classification of geographical origins and authenticity of C. pilosula samples was accomplished by using TQ Analyst (Thermo Fisher, USA) with DA method. Calibration model of quantitative analysis of polysaccharide content in C. pilosula was built with Matlab 2015a and PLS_toolbox (Mathworkers Inc., USA).

\subsection{Phenol-sulfuric acid method}

\subsubsection{Reference solutions}

The obtained anhydrous glucose $(0.6031 \mathrm{~g})$ was dissolved in a small amount of distilled water, and then the solution was diluted to $100 \mathrm{~mL}$. An aliquot of $10 \mathrm{~mL}$ solution was taken out and diluted 10 times, and the concentration of reference solution was $0.6031 \mathrm{mg} / \mathrm{mL}$.

\subsubsection{Standard curve}

Seven reference solutions with different volumes $(0$, $0.2,0.4,0.6,0.8,1.0$, and $1.2 \mathrm{~mL}$ ) were added into seven tubes with caps, respectively. Each solution

Table 1. Information on samples.

\begin{tabular}{lcccc}
\hline Sample & Name & Trade name & No. & Origin \\
\hline Authenticity & Codonopsis pilosula (Franch.) Nannf. & Baitiao & $1-15$ & Gansu \\
& & Dongdang & $16-30$ & Northeast \\
& Cudang & $31-45$ & Shanxi \\
& Codonopsis tangshen Oliv. & Chuandang & $46-57$ & Sichuan \\
& Codonopsis pilosula Nannf. var. & Wendang & $58-72$ & Gansu \\
& modesta (Nannf.) L. T Shen & & & \\
Fake & Codonopsis pilosula (Franch.) Nannf. & Sulfur-Baitiao & $73-87$ & Gansu \\
& Radix Stellariae & Radix Stellariae & $88-102$ & Ningxia Neimenggu \\
& & & & (Inner Mongolia) \\
\hline
\end{tabular}




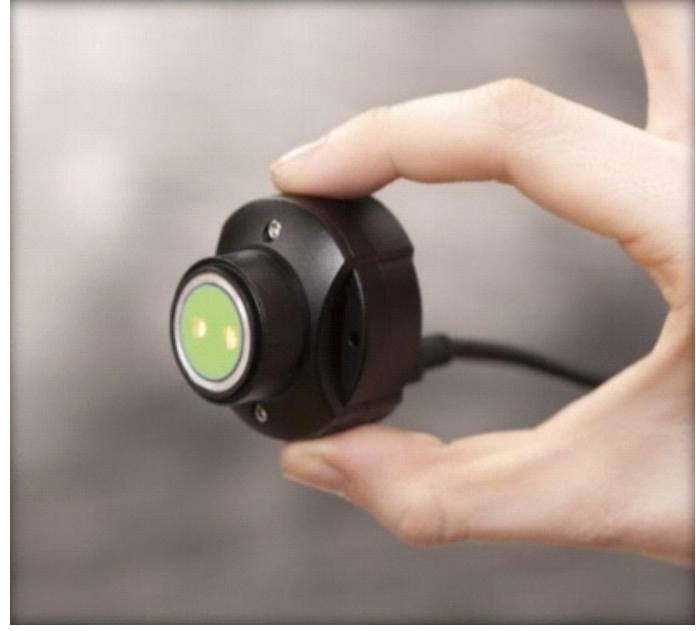

Fig. 1. Micro NIR 1700 spectrometer.

was diluted to $2.0 \mathrm{~mL}$ with distilled water. The $5 \%$ phenol solution $(1 \mathrm{~mL})$ and concentrated sulfuric acid $(5 \mathrm{~mL})$ were added to each tube. Then the tubes were closed, shaken well, and incubated for $15 \mathrm{~min}$ at $40^{\circ}$. All of the tubes were allowed to cool down to room temperature before reading their absorption at $490 \mathrm{~nm}$ in a Microplate Reader (Awareness, USA). Then, standard curve was drawn between absorption $(A)$ and concentration $(C)$ of anhydrous glucose by linear regression.

\subsubsection{Sample solutions}

No. 1-87 samples were used for quantitative analysis of polysaccharide content in C. pilosula. Firstly, sample powder $(0.5 \mathrm{~g})$ and $80 \%$ ethanol $(25 \mathrm{~mL})$ were added into $50 \mathrm{~mL}$ centrifuge tube. The ultrasonic method was used for extraction for about $25 \mathrm{~min}$. The supernatant was collected after centrifugation. Secondly, water $(28 \mathrm{~mL})$ was added into the tube containing residue and ultrasonic extraction was then performed for 10 min. The supernatant was collected after centrifugation. Thirdly, these supernatants were merged together and then diluted to $100 \mathrm{~mL}$.

\subsubsection{Determining the polysaccharide concentration of sample solution}

Liquid $(0.2 \mathrm{~mL})$ was taken out from each sample solution, and then absorption could be measured following the above phenol-sulfuric acid method. Each sample was recorded three times and then averaged for subsequent analysis. Finally, the concentration of polysaccharide in sample solution was calculated according to the standard curve.

\subsection{Discriminant model for authenticity}

In 102 samples, 72 authentic samples and 30 fake $C$. pilosula were used to establish a discriminant model by DA method. Four kinds of spectral pretreatment methods, including standard normal variate (SNV) transformation, Savitzky-Golay (SG) smoothing, first derivative with SG 7 points filter (1 st + SG7) and SNV+SG 7, were compared to choose the optimal one according to model performance.

The model established with full spectra region (908-1676 nm) and optimal spectral pretreatment method were also further assessed by principle component analysis (PCA).

\subsection{Classification model for geographical origins}

Forty-five batches of $C$. pilosula (Franch.) Nannf. were collected to establish DA model from three areas including Gansu province (trade name: Baitiao, No. 1-15), Northeast of China (trade name: Dongdang, No. 16-30), and Shanxi province (trade name: Ludang, No. 31-45). The four pretreatment methods including SNV, SG 7, 1 st + SG 7, and $\mathrm{SNV}+1 \mathrm{st}+\mathrm{SG} 7$ were compared to choose the best one according to the performance of the model.

PCA was used to evaluate the DA model for discrimination of sample geographical origin with full spectral region and optimal spectral pretreatment method.

\subsection{Quantitative analysis}

At first, insignificant spectral region should be eliminated by analyzing the raw spectra. Then, the raw spectra were pretreated by multiplicative scatter correction (MSC), SNV, 1 st, and 1 st + SG 7 as well as no pretreatment, respectively. The calibration set was established and the optimal pretreatment method was chosen according to the model performance, which was based on the values of $R_{c}, R_{p}$, RMSECV, and RMSEP.

Additionally, three methods were investigated to select the optimal spectral region, including competitive adaptive reweighted sampling (CARS)PLS, interval PLS (iPLS), and successive projections algorithm (SPA). 
A quantitative model of calibration set with optimal pretreatment method and spectral region was used to predict the polysaccharide concentration of validation set.

\section{Results and Discussion}

\subsection{Raw spectra of samples}

Figure 2 showed the raw spectra of samples. From Fig. 2 we can see that 102 samples of spectral absorption trend had serious overlapping. It was difficult to get information directly from the peak position and characteristic peak information. Only by using chemometric methods to deal with the spectra, the effective information could be highlighted out.

Figure 3 showed the spectra pretreated by 1 st + SG 7 of 102 samples. There were two sharp peaks that could be observed in regions of $1165 \mathrm{~nm}$ and $1425 \mathrm{~nm}$ from the raw and preprocessed spectra. The absorbance in the range of $1165 \mathrm{~nm}$ was attributed by the third overtone of $\mathrm{C}-\mathrm{H}$ bands. Meanwhile, the waveband of $1425 \mathrm{~nm}$ was associated with the second overtone stretch and deformation vibration of $\mathrm{O}-\mathrm{H}$ bands.

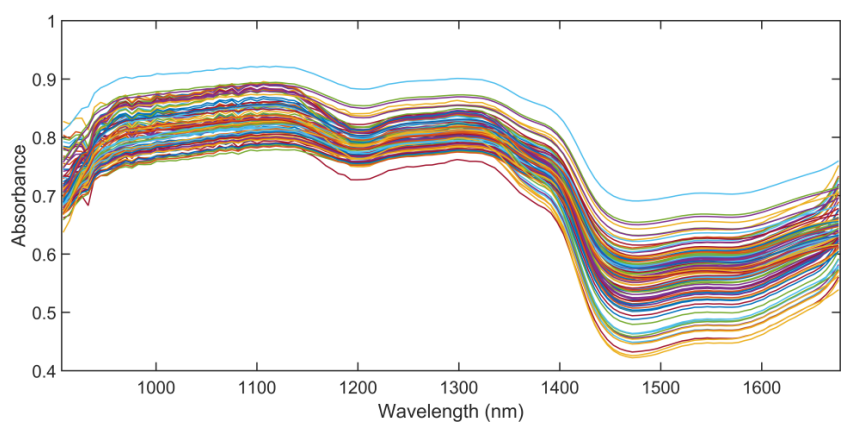

Fig. 2. Raw spectra of C. pilosula samples.

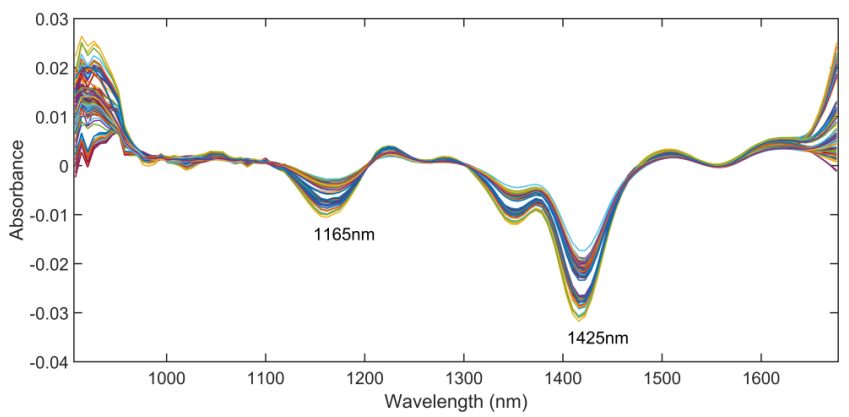

Fig. 3. Spectra of samples pretreated by 1 st + SG 7 .

\subsection{Division of sample sets}

Figure 4 showed the scores of all the samples of seven classes with PCA. The red circle represented the sample in calibration set, whereas the blue plus sign represented the sample in the validation set. It showed that samples of both sets had uniform distribution, which means that two sample sets were divided reasonably and the calibration set could be used to construct quantitative model.

\subsection{Results of discriminant model for authenticity}

The results of different spectral pretreatment methods were shown in Table 2, which indicated the discriminant performances. The optimal spectral pretreatment method was SNV+SG 7 method. The model based on full spectral region combined with SNV+SG 7 had a better predictive ability with $100 \%$ accuracy, which could be useful for quickly detecting the authenticity of $C$. pilosula.

The performance of the discriminant model was also illustrated in the 3-D score plot of the first three principal components (Fig. 5). As shown in Fig. 5, there were clear differences between the two-class samples.

\subsection{Classification model of geographical origins}

Results of classification model of geographical origins were shown in Table 3. From Table 3, the optimal discrimination performance of calibration set was based on the SNV+1st+SG 7 method. The predictive accuracy was up to $100 \%$ based on the optimal model with full spectra region and $\mathrm{SNV}+\mathrm{SG} 7$ method. The optimal model could be used for rapidly detecting the geographical origins of $C$. pilosula.

To further assess the performance of the DA model for classifying samples according to geographical origins, PCA was also employed. From the 3 -D score plot of PCA (Fig. 6), there were distinct gaps between the samples from three geographical origins.

\subsection{Results of phenol-sulfuric acid method}

\subsubsection{Polysaccharide content of samples}

The linear regression formulation was as follows: $A=$ $25.08 C+0.0028 \quad\left(R^{2}=0.9998\right)$. Standard curve 


\section{J. Wang et al.}

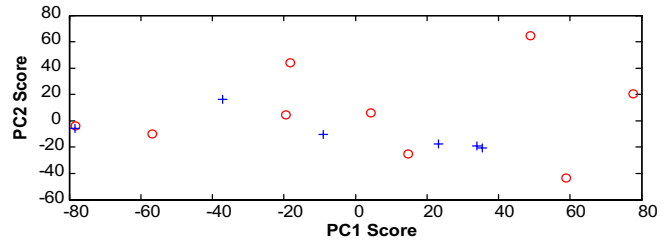

(a)

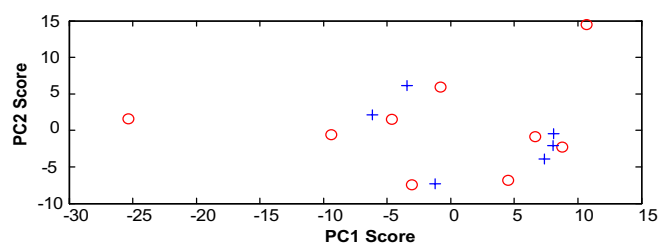

(c)

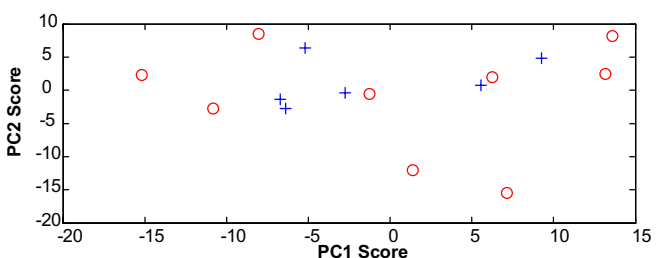

(e)

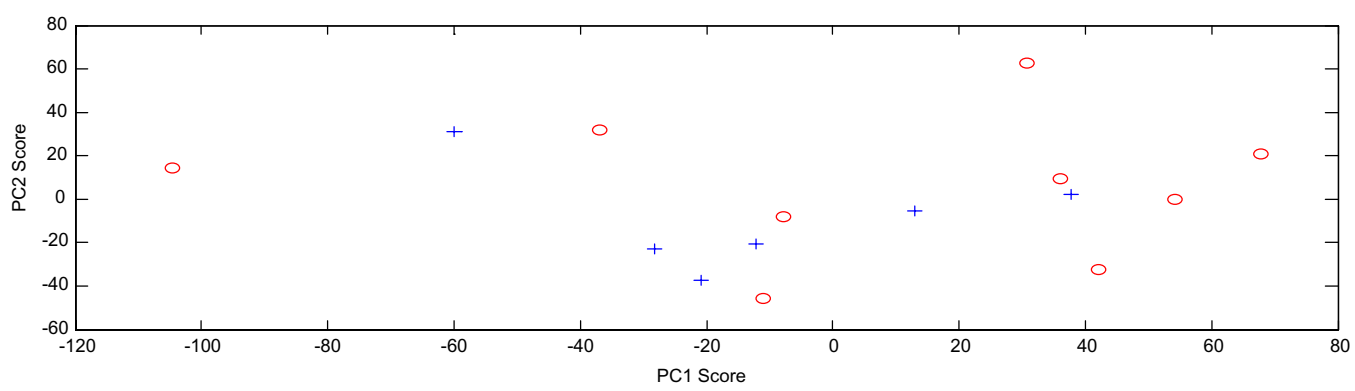

(g)

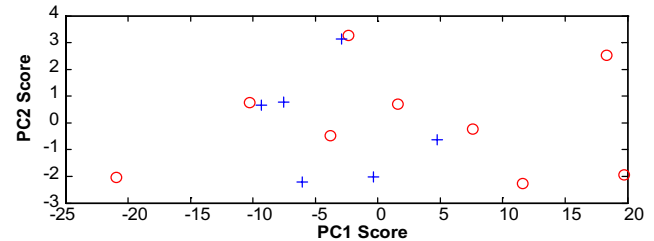

(b)

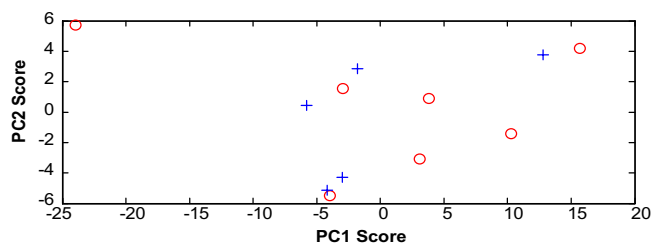

(d)

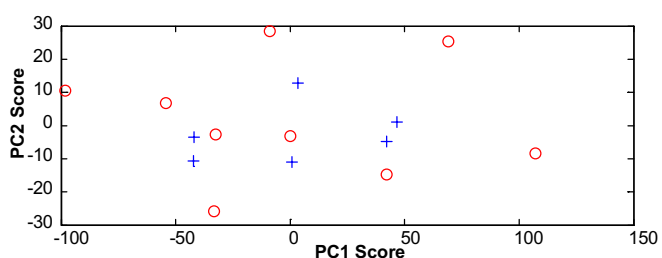

(f)

Fig. 4. The scores of all the seven samples with PCA. (a) Baitiao (Gansu); (b) Dongdang (Northeast); (c) Ludang (Shanxi); (d) Chuandang (Sichuan); (e) Wendang (Gansu); (f) Sulfur Baitiao (Gansu); and (g) Radix Stellariae (Ningxia).

Table 2. Results of models based on different spectral pretreatment methods.

\begin{tabular}{lccrrrrr}
\hline & \multicolumn{3}{c}{ Calibration } & & \multicolumn{3}{c}{ Validation } \\
\cline { 2 - 3 } Pretreatment method & No. & False no. & $\begin{array}{r}\text { Accuracy } \\
\text { rate }(\%)\end{array}$ & & No. & False no. & $\begin{array}{r}\text { Accuracy } \\
\text { rate (\%) }\end{array}$ \\
\hline No-pretreated & 61 & 7 & 88.5 & 41 & 4 & 90.2 \\
SNV & 61 & 0 & 100.0 & 41 & 1 & 97.6 \\
S-G 7 & 61 & 4 & 95.1 & 41 & 2 & 95.1 \\
1st+S-G 7 & 61 & 2 & 93.4 & 41 & 0 & 100.0 \\
SNV+S-G 7 & 61 & 0 & 100.0 & 41 & 0 & 100.0 \\
\hline
\end{tabular}


Table 3. Results of classification model of geographical origins based on different spectral pretreatment methods.

\begin{tabular}{lccccccc}
\hline & \multicolumn{3}{c}{ Calibration } & & \multicolumn{3}{c}{ Validation } \\
\cline { 2 - 3 } Pretreatment method & No. & False no. & $\begin{array}{c}\text { Accuracy } \\
\text { rate }(\%)\end{array}$ & & No. & False no. & $\begin{array}{c}\text { Accuracy } \\
\text { rate (\%) }\end{array}$ \\
\hline No-pretreated & 27 & 4 & 85.2 & & 18 & 3 & 83.3 \\
SNV & 27 & 2 & 92.6 & & 18 & 0 & 100.0 \\
SG 7 & 27 & 3 & 88.9 & 18 & 2 & 88.9 \\
1st+SG 7 & 27 & 0 & 100.0 & 18 & 1 & 94.4 \\
SNV+1st+SG 7 & 27 & 0 & 100.0 & 18 & 0 & 100.0 \\
\hline
\end{tabular}

showed that it was a strong linear relationship between $A$ (absorbance) and $C$ (concentration), which met the requirement of analysis.

\subsection{Quantitative model based on NIRS \\ 3.6.1. Elimination of insignificant spectral region}

As shown in Figs. 1 and 2, there were some noises in the spectral range of $908-1140 \mathrm{~nm}$ waveband, resulting in little useful absorption information in this range. Therefore, the waveband should be discarded before the calibration process. To confirm the effectiveness of the band selection, models were established with different wavebands. As shown in Table 4 , the values of $R_{c}, R_{p}$, RMSEC, and RMSEP were $0.9428,0.9275,3.4168$, and $3.8214 \mathrm{~g} / \mathrm{mL}$, respectively, and the quantitative model was constructed in the range of 1140-1676 nm waveband.

\subsubsection{Selection of spectral pretreatment method}

According to the aforementioned results (Table 4), 1140-1676 nm wavebands were used to select the optimal pretreatment method. ${ }^{25,26}$ As shown in Table 5, it is indicated that the MSC method had a better result compared with other methods. The values of RMSECV and RMSECP were minimal, ensuring the accuracy of the prediction results.

\subsubsection{NIR waveband selection}

With the optimal pretreatment method, the spectral region was further selected based on the performances of PLS models. Table 6 showed the results of PLS models with different variable selection methods. When iPLS and SPA were adopted to select wavebands, the results of model became better. Meanwhile, the number of variables also

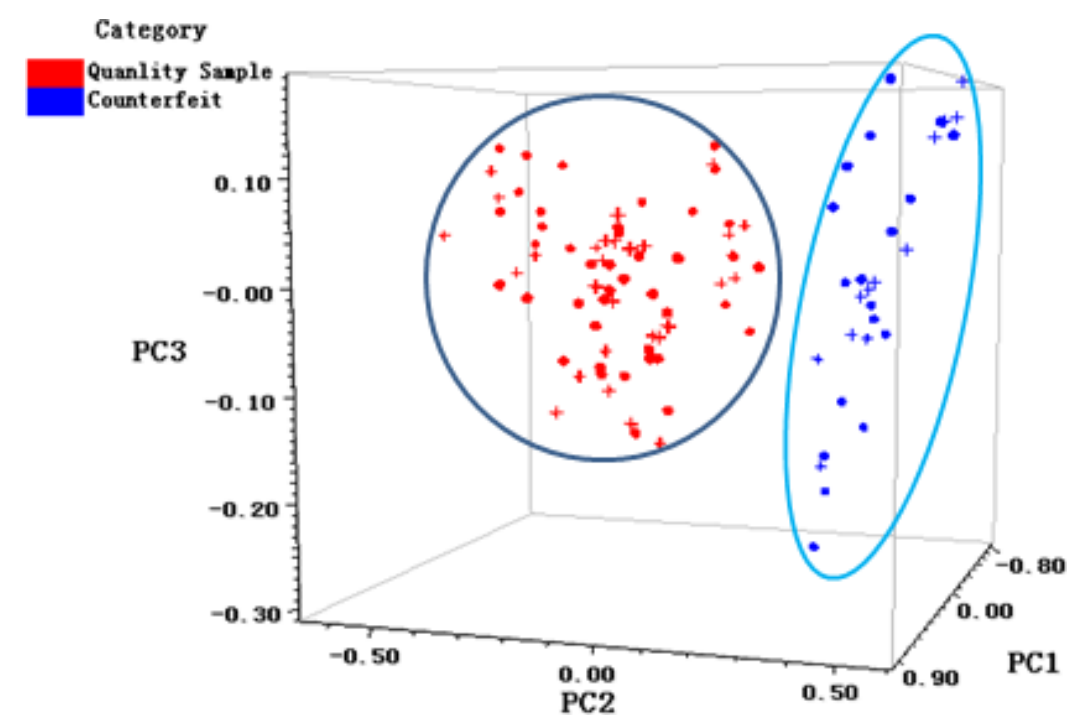

Fig. 5. Plot of PCA scores (PC1 vs PC2 vs PC3) of samples (red dot represents the authentic sample, and blue dot represents the fake sample). Circular dot represents the calibration set sample, and crossed dot represents the validation set samples. 
J. Wang et al.

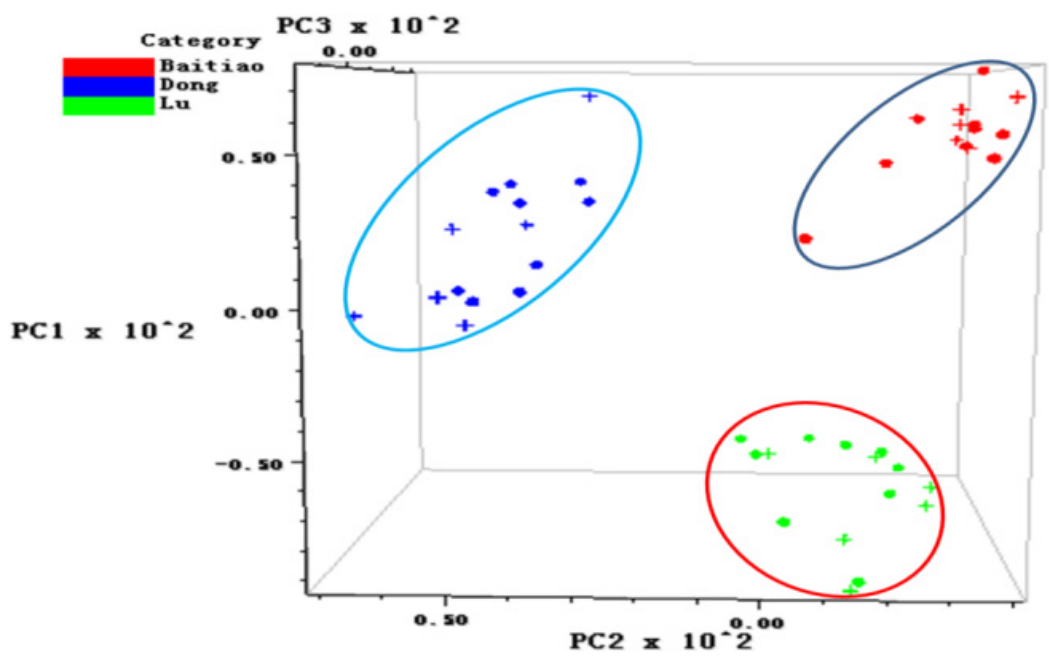

Fig. 6. Plots of PCA scores (PC1 vs PC2 vs PC3) of three geographical classes samples. Red represents the Baitiao samples (Gansu); blue represents the Ludang samples (Shanxi); and green represents the Dongdang samples (Northeast).

decreased. According to the results (Table 6), the iPLS method was superior to SPA. So the wavebands of 1465-1521 nm and 1620-1645 nm selected by the iPLS method were used as the optimal spectral region. The results of selected variable were shown in Fig. 7.

\subsubsection{Prediction capability of $P L S$ quantitative model}

In conclusion, selecting MSC as the spectral pretreatment method, iPLS method was applied to choose the spectral region ranging from $1465 \mathrm{~nm}$ to $1521 \mathrm{~nm}$ and from $1620 \mathrm{~nm}$ to $1645 \mathrm{~nm}$. The results

Table 4. Results before and after elimination of insignificant waveband.

\begin{tabular}{lcccccc}
\hline Wave band (nm) & PCs & $R_{c}$ & $R_{p}$ & RMSEC $(\mathrm{g} / \mathrm{mL})$ & RMSECV $(\mathrm{g} / \mathrm{mL})$ & RMSEP $(\mathrm{g} / \mathrm{mL})$ \\
\hline Whole band & 7 & 0.9267 & 0.894 & 3.832 & 5.034 & 4.524 \\
$908-1140$ & 7 & 0.8730 & 0.477 & 5.534 & 7.052 & 7.583 \\
$1140-1676$ & 6 & 0.9428 & 0.928 & 3.417 & 3.762 & 3.821 \\
\hline
\end{tabular}

Table 5. Results of different spectral pretreatment methods for quantitative model.

\begin{tabular}{lcccccc}
\hline Pretreatment & PCs & $R_{c}$ & $R_{p}$ & RMSEC $(\mathrm{g} / \mathrm{mL})$ & RMSECV $(\mathrm{g} / \mathrm{mL})$ & RMSEP $(\mathrm{g} / \mathrm{mL})$ \\
\hline No pretreatment & 6 & 0.9428 & 0.9275 & 3.417 & 3.762 & 3.821 \\
MSC & 7 & 0.9630 & 0.9515 & 2.532 & 2.887 & 3.138 \\
SNV & 7 & 0.9576 & 0.9485 & 2.608 & 2.954 & 3.261 \\
1 st & 6 & 0.9369 & 0.8961 & 3.755 & 4.654 & 4.895 \\
1st+SG 7 & 7 & 0.9444 & 0.9128 & 3.044 & 3.673 & 3.593 \\
\hline
\end{tabular}

Table 6. Results of different spectral regions by various variable selection methods.

\begin{tabular}{lccccccc}
\hline Methods & Variables no. & PCs & $R_{c}$ & $R_{p}$ & RMSEC $(\mathrm{g} / \mathrm{mL})$ & RMSECV $(\mathrm{g} / \mathrm{mL})$ & RMSEP $(\mathrm{g} / \mathrm{mL})$ \\
\hline $1140-1676 \mathrm{~nm}$ & 87 & 7 & 0.9630 & 0.9515 & 2.532 & 2.887 & 3.138 \\
iPLS & 16 & 8 & 0.9775 & 0.9602 & 2.240 & 2.496 & 2.734 \\
CARS-PLS & 11 & 5 & 0.9572 & 0.9483 & 3.796 & 2.053 & 3.875 \\
SPA & 20 & 6 & 0.9715 & 0.9558 & 2.573 & 2.887 & 3.138 \\
UVE & 25 & 6 & 0.9603 & 0.9420 & 2.201 & 2.502 & 2.613 \\
\hline
\end{tabular}


Table 7. Results of validation set predicted by optimal PLS quantitative model (\%).

\begin{tabular}{lcccccrr}
\hline No. & $\begin{array}{c}\text { True value } \\
(\mathrm{g} / \mathrm{mL})\end{array}$ & $\begin{array}{c}\text { Predictive } \\
\text { value }(\mathrm{g} / \mathrm{mL})\end{array}$ & $\begin{array}{c}\text { Absolute } \\
\text { error }(\mathrm{g} / \mathrm{mL})\end{array}$ & No. & $\begin{array}{c}\text { True value } \\
(\mathrm{g} / \mathrm{mL})\end{array}$ & $\begin{array}{c}\text { Predictive } \\
\text { value }(\mathrm{g} / \mathrm{mL})\end{array}$ & $\begin{array}{r}\text { Absolute } \\
\text { error }(\mathrm{g} / \mathrm{mL})\end{array}$ \\
\hline 1 & 32.40 & 32.74 & 0.34 & 46 & 41.92 & 40.70 & -1.22 \\
6 & 34.45 & 36.17 & 1.72 & 47 & 46.33 & 44.60 & -1.73 \\
10 & 33.04 & 32.74 & -0.30 & 50 & 37.79 & 36.66 & -1.13 \\
12 & 31.41 & 32.65 & 1.24 & 51 & 44.52 & 44.38 & -0.14 \\
13 & 33.54 & 33.95 & 0.41 & 56 & 33.95 & 35.75 & 1.80 \\
14 & 30.51 & 33.47 & 2.96 & 59 & 41.01 & 38.28 & -2.73 \\
17 & 47.85 & 48.96 & 1.11 & 63 & 40.63 & 38.42 & -2.21 \\
18 & 29.26 & 30.44 & 1.18 & 65 & 36.52 & 37.88 & 1.36 \\
25 & 48.41 & 48.40 & -0.01 & 67 & 35.23 & 36.28 & 1.05 \\
27 & 43.15 & 42.32 & -0.83 & 70 & 37.94 & 40.38 & 2.44 \\
28 & 38.86 & 40.28 & 1.42 & 71 & 42.22 & 46.84 & 4.62 \\
29 & 40.10 & 42.51 & 2.41 & 74 & 36.06 & 35.32 & -0.74 \\
32 & 18.25 & 22.91 & 4.66 & 75 & 28.49 & 30.73 & 2.24 \\
36 & 25.32 & 26.00 & 0.68 & 82 & 36.50 & 36.27 & -0.23 \\
38 & 40.43 & 40.58 & 0.15 & 84 & 38.87 & 39.77 & 0.90 \\
39 & 32.04 & 33.17 & 1.13 & 85 & 33.38 & 31.76 & -1.62 \\
40 & 33.34 & 32.31 & -1.03 & 86 & 29.99 & 31.59 & 1.60 \\
44 & 38.32 & 40.80 & 2.48 & & & & \\
\hline
\end{tabular}

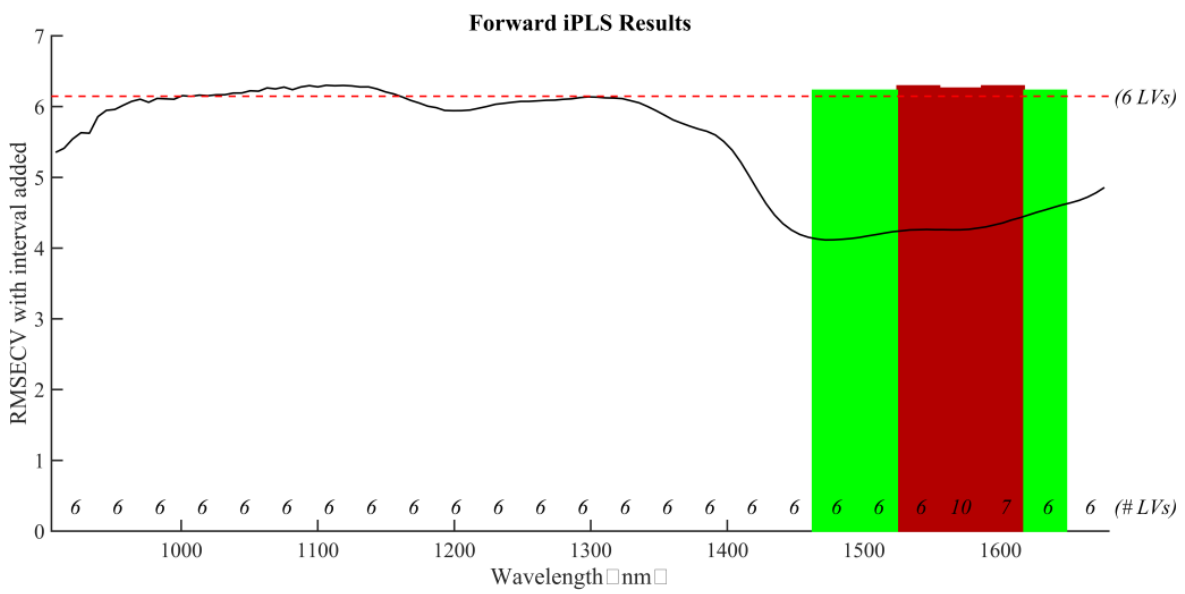

Fig. 7. The result of variable selection.

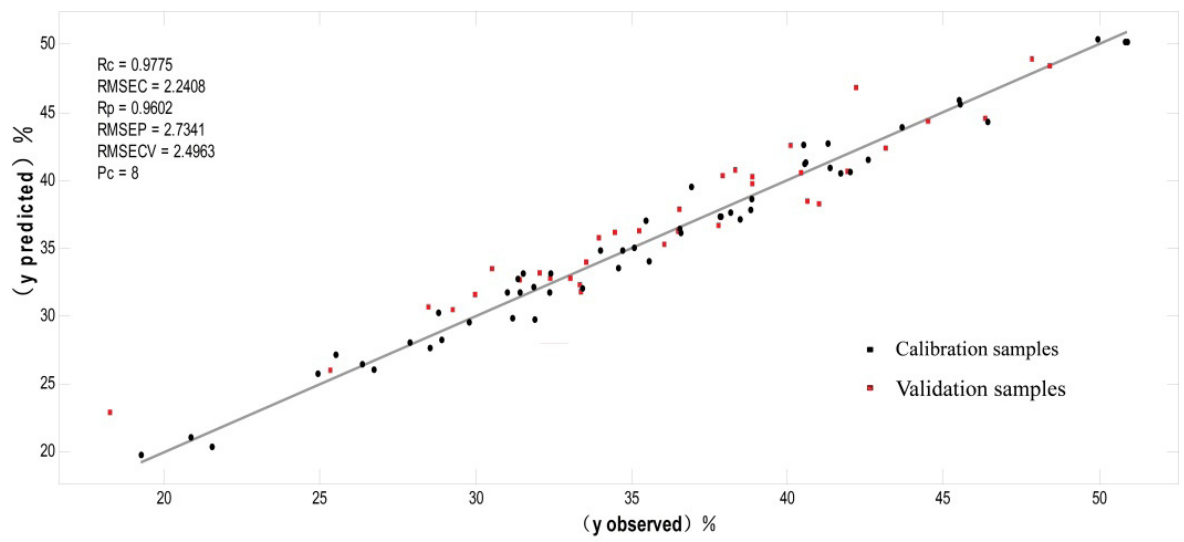

Fig. 8. Correlation diagram of polysaccharide content. 
of quantitative model established with PLS regression showed that the values of $R_{p}$, RMSECV, and RMSEP were, respectively, 0.9602, 2.4963, and $2.7341 \mathrm{~g} / \mathrm{mL}$, which indicated that the established model had higher predication ability for predicting the concentration of polysaccharide content in $C$. pilosula. Detailed results were shown in Table 7 and Fig. 8.

\section{Conclusion}

In this study, qualitative model was established for geographical origin and authenticity discrimination of C. pilosula with Micro NIR 1700 spectrometer successfully. The optimal DA model had a predictive ability with $100 \%$ accuracy. In addition, an NIR method was also developed for the determination of polysaccharide content in C. pilosula. The calibration model was validated with satisfactory $R_{p}$, RMSECV, and RMSEP values, which were $0.9602,2.4963$, and $2.7341 \mathrm{~g} / \mathrm{mL}$, respectively. Due to the rapid, accurate, and robust properties, the qualitative and quantitative models based on Micro NIR 1700 spectrometer could be successfully used for monitoring the quality of C. pilosula. The presented method provided a promising tool for the quality of raw TCM material evaluation and could be potential application for solving the similar issues. Compared with general NIR instruments, micro instrument such as Micro NIR 1700 spectrometer is much cheaper, convenient, and portable, which would promote the application of micro NIR spectrometer, especially in the quality control of TCM.

\section{Acknowledgments}

This work was supported by the Project of Shandong Province (No. 2012CX20203) and the Major Special Project of National Scientific Instruments and Equipment Development (No. 2014YQ47037709).

\section{References}

1. Y. Jiang, B. David, P. F. Tu, Y. Barbin, "Recent analytical approaches in quality control of traditional Chinese medicines - A review," Anal. Chim. Acta 657, 9-18 (2010). doi: 10.1016/j.aca.2009.10.024.

2. Y. X. Sun, J. C. Liu, "Structural characterization of a water-soluble polysaccharide from the roots of
Codonopsis pilosula and its immunity activity," Int. J. Biol. Macromol. 43, 279-282 (2008). doi: 10.1016/j.ijbiomac.2008.06.009.

3. Y. F. Zou, X. F. Chen, W. Y. Yang, S. Liu, "Response surface methodology for optimization of the ultrasonic extraction of polysaccharides from Codonopsis pillosula Nannf. var. modesta L. T. Shen," Carbohydr. Polym. 84, 503-508 (2011). doi: 10.1016/j.carbpol.2010.12.013.

4. Z. T. Wang, T. B. Ng, H. W. Yeung, G. J. Xu, "Immunomodulatory effect of a polysaccharideenriched preparation of Codonopsis pilosula roots," Gen. Pharmacol. 27, 1347-1350 (1996). doi: 10.1016/S0306-3623(96)00084-5.

5. G. Z. Liu, D. G. Cai, S. Shao, "Studies on the chemical constituents and pharmacological actions of Dangshen, Codonopsis pilosula (Franch.) Nannf," J. Tradit. Chin. Med. 8, 41-47 (1988). View Record in Scopus|Citing articles (12).

6. H. Luo, S. Lin, F. Ren, L. Wu, L. Chen, Y. Sun, "Antioxidant and antimicrobial capacity of Chinese medicinal herb extracts in raw sheep meat," J. Food Prot. 70, 1440-1445 (2007). CrossRef | View Record in Scopus | Citing articles (31).

7. B. E. Shan, Y. Yoshida, T. Sugiura, U. Yamashita, "Stimulating activity of Chinese medicinal herbs on human lymphocytes in vitro," Int. J. Immunopharmacol. 21, 149-154 (1999).

8. G. Cuesta, N. Suarez, M. I. Bessio, F. Ferreira, H. Massaldi, "Quantitative determination of pneumococcal capsular polysaccharide serotype 14 using a modification of phenol-sulfuric acid method," J. Microbiol. Methods 52, 69-73 (2003). doi: 10.1016/ S0167-7012(02)00151-3.

9. J. Luypaert, D. L. Massart, Y. Vander Heyden, "Near-infrared spectroscopy applications in pharmaceutical analysis," Talanta 72, 865-883 (2007). doi: $10.1016 /$ j.talanta.2006.12.023.

10. J. W. Braga, A. D. Araci, I. S. Martinsb, "Determination of viscosity index in lubricant oils by infrared spectroscopy and PLSR," Fuel 120, 171-178 (2014). doi: 10.1016/j.fuel.2013.12.017.

11. D. H. Pan, G. Crull, S. Yin, J. Grosso, "Low level drug product API form analysis-Avalide tablet NIR quantitative method development and robustness challenges," J. Pharmaceut. Biomed. Anal. 89, 268275 (2014). doi: 10.1016/j.jpba.2013.11.011.

12. J. Duan, Y. Huang, Z. H. Li, B. Zheng, Q. Q. Li, Y. M. Xiong, L. J. Wu, S. G. Min, "Determination of 27 chemical constituents in Chinese south west tobacco by FT-NIR spectroscopy," Indus. Crop Prod. 40, 21-26 (2012). doi: 10.1016/j.indcrop.2012.02.040.

13. Q. Dong, H. C. Zang, A. H. Liu, G. L. Yang, C. X. Sun, L. Y. Sui, P. Wang, L. Li, "Determination of molecular weight of hyaluronic acid by near-infrared 
spectroscopy," J. Pharmaceut. Biomed. Anal. 53, 274-278 (2010). doi: 10.1016/j.jpba.2010.05.031.

14. O. Y. Rodionova, L. P. Houmoller, A. L. Pomerantsev, P. Geladi, J. Burger, V. L. Dorofeyev, A. P. Arzamastsev, "NIR spectrometry for counterfeit drug detection: A feasibility study," Anal. Chim. Acta 549, 151-158 (2005). doi: 10.1016/j.aca.2005.06.018.

15. X. J. Duan, D. L. Zhang, L. Nie, H. C. Zang, "Rapid discrimination of geographical origin and evaluation of antioxidant activity of Salvia miltiorrhiza var. alba by Fourier transform near infrared spectroscopy," Spectrochim. Acta A Mol. Biomol. Spectrosc. 122, 751-757 (2014). doi: 10.1016/j.saa.2013. 12.003.

16. P. W. Z. Yu, "Species authentication and geographical origin discrimination of herbal medicines by near infrared spectroscopy: A review," J. Pharmaceut. Anal. 5, 227-284 (2015). doi: 10.1016/j. jpha.2015.04.001.

17. F. Wang, W. Jiang, C. Li, H. Zhang, L. Nie, L. Li, P. Wang, H. C. Zang, "Application of near infrared spectroscopy in monitoring the moisture content in freeze-drying process of human coagulation factor VIII," J. Innov. Opt. Health Sci. 8, 150034 (2015). doi: $10.1142 / \mathrm{S} 1793545815500340$.

18. P. Wang, H. Zhang, H. L. Yang, L. Nie, H. C. Zang, "Rapid determination of major bioactive isoflavonoid compounds during the extraction process of kudzu (Pueraria lobata) by near-infrared transmission spectroscopy," Spectrochim. Acta A Mol. Biomol. Spectrosc. 137, 1403-1408 (2015). doi: 10.1016/j.saa.2014.09.002.

19. T. Hu, H. L. Yang, Q. Tang, H. Zhang, L. Nie, L. Lian, J. F. Wang, W. Jiang, F. Wang, H. C. Zang, "Absolutely nondestructive discrimination of Huoshan Dendrobium nobile species with micro nearinfrared (NIR) spectrometer engine," Spectrosc. Spect. Anal. 34, 2808-2814 (2014). doi: http://dx. doi.org/10.3964/j.issn.1000-0593(2014)10-2808-07.
20. X. Pan, F. Li, Z. Wu, Z. Lin, "Near infrared spectroscopy model development and variable importance in projection assignment of particle size and lobetyolin content of Codonopsis radix," J. Near Infrared Spec. 23, 461-480 (2011).

21. B. Li, Y. Wei, H. Duan, L. Xi, "Discrimination of the geographical origin of Codonopsis pilosula, using near infrared diffuse reflection spectroscopy coupled with random forests and k-nearest neighbor methods," Vib. Spectrosc. 62, 17-22 (2012).

22. B. Li, Y. H. Wei, H. G. Duan, L. L. Xi, X. N. Wu, "Discrimination of the geographical origin of Codonopsis pilosula using near infrared diffuse reflection spectroscopy coupled with random forests and k-neighbor methods," Vib. Spectrosc. 62, 17-22 (2012). doi: 10.1016/j.vibspec.2012.05.001.

23. O. Galtier, Y. Abbas, Y. L. Dréau, C. Rebufa, J. Kister, J. Artaud, N. Dupuy, "Comparison of PLS1DA, PLS2-DA and SIMCA for classification by origin of crude petroleum oils by MIR and virgin olive oils by NIR for different spectral regions," Vib. Spectrosc. 55, 132-140 (2011). doi: 10.1016/j. vibspec.2010.09.012.

24. Q. S. Chen, J. W. Zhao, C. H. Fang, D. M. Wang, "Feasibility study on identification of green, black and Oolong teas using near-infrared reflectance spectroscopy based on support vector machine (SVM)," Spectrochim. Acta A Mol. Biomol. Spectrosc. 66, 568574 (2007). doi: 10.1016/j.saa.2006.03.038.

25. W. L. Li, Zh. W. Cheng, Y. F. Wang, H. B. Qu. "Feasibility research on noninvasive analysis of Tanreqing injection with near infrared spectroscopy," $J$. Near Infrared Spectrosc. 20, 667-674 (2012).

26. H. F. Han, L. Zhang, Y. Zhang, W. L. Li, H. B. Qu, "Rapid determination of total flavonoids, total saponins, and soluble solid content in Fufang Ejiao Syrup by NIRS," Chin. Tradit. Herb. Drugs 44, 2397-2403 (2013). 